\title{
Laser cleaning for rust removal on mild steel: An experimental study on surface characteristics
}

\author{
Vishnu Narayanan, Ramesh K Singh and Deepak Marla \\ Department of Mechanical Engineering, IIT Bombay, Maharashtra, India
}

\begin{abstract}
Laser cleaning is a relatively new and promising industrial cleaning method. Despite its potential as an alternative to conventional de-rusting methods, very few studies have been done on rust removal. The current study is aimed at investigating experimentally the effect of various important laser parameters on properties of surface after laser processing. Rusted mild steel samples were irradiated with moving pulsed laser beam. Parameters like scan speed, number of passes, etc. were varied and the change in properties such as surface profile, roughness, hardness, etc. of the processed surface were observed along with depth of removal. The depth of removal was found to increase with an increase in laser power, and number of scans but decreased with an increase in scan speed. The surface got discoloured and deformed at lower speeds and higher powers. The surface roughness after processing hardly showed any variation with the initial roughness values before cleaning but depended on the scanning path of the laser. The microhardness values after laser processing varied with path traced by the laser beam which points to microstructure changes in the samples due to laser processing.
\end{abstract}

\section{Introduction}

Laser beams can be used for removal of paint [1], rust [2], grease [3], etc. from surfaces and also for cleaning of dies used in molding [4]. This method is non-contact as well as non-destructive. Laser cleaning doesn't need any abrasive or chemical media. The suitable laser type and optimum parameters are different for each application [5]. The actual physical process depend on the operating conditions and various parameters which determine the outcome of the cleaning process. So these parameters have to be chosen carefully to get the desired outcome.

Several previous studies had been done to identify the optimum working conditions for laser cleaning of rust. A lot of them focussed on cleaning and conservation of archeological artefacts [6]. Other works focussed on finding the threshold fluence for a particular laser equipment, optimum working conditions for certain application [7] or to find the mechanisms behind the cleaning process [7]. Koh and Sarady [6] conducted experiments to study the ideal wavelength and fluence for laser cleaning of corroded artefacts. It was concluded that TEA $\mathrm{CO}_{2}$ laser was better compared to Nd:YAG laser since risk of damage was higher with Nd:YAG laser. Meja et al. [7] conducted experiments to study effect of wavelength, fluence, pulse repetition rate and environment of laser cleaning of rust from iron. Daurelio et al. [2] studied the rust removal rate for different fluence and pulse repetition rate. Wang et al. [8] conducted experimental study on de-rusting of steel blocks using Nd:YAG laser. The parameters for complete removal of rust was studied and the change in roughness, hardness and corrosion resistance with change in fluence were studied. Chen et al. [9] studied the effectiveness of fibre laser in removal of rust, grease, etc. and concluded that laser cleaning is a good alternative for conventional methods of surface preparation in ship building. Ali et al. [3] studied the change in depth of removal, roughness hardness, etc. with change in peak pulse power and pulse repetition rate.

The literature survey pointed out that the surface damage and type of morphological changes that happen during laser cleaning of rust has not been adequately studied. In addition, effect of each laser parameter on the surface characteristics after laser processing has not been reported properly. In order to obtain desired surface properties and avoid damage, a knowledge on the relative importance of each parameter on surface characteristics post laser processing will be helpful to choose the optimum parameters.

The objective of this study was to conduct experiments in order to observe the change in surface roughness and the surface damage with the change in laser parameters during laser cleaning of rust. This will help in predicting the optimum conditions for laser cleaning of rust. In this work, rusted mild steel samples are de-rusted with nanosecond pulsed laser beam of wavelength $1064 \mathrm{~nm}$. The samples were scanned with the laser and the effects like depth of material removal, change in surface properties, and morphology after laser irradiation were studied.

\section{Experiments}


In this study, a nanosecond pulsed laser with an average power of $30 \mathrm{~W}$ and a wavelength of $1064 \mathrm{~nm}$, with a fibre delivery system was used. It has a pulse width of $80 \mathrm{~ns}$ and pulse repetition rate was kept at $50 \mathrm{kHz}$ for all the experiments. It was equipped with a galvo-scanner coupled with an F-theta lens, using which the laser was scanned on the surface of samples.

Experiments were conducted to study the effect of scan speed and number of passes on the surface of rusted and pure mild steel surfaces. When rust is removed by laser cleaning, the pure steel layer beneath the rust layer would also get subjected to laser irradiation as process nears completion. Therefore, it is important to know the adverse effects of process parameters that are optimal for effective rust layer removal. In order to understand the heating effects occurring underneath the rust layer and quantify the damage, experiments were also conducted on pure steel samples. Lines were scanned on the mild steel samples at different speeds keeping all other parameters same and the samples were analysed using Zeta optical profile analyser and the effects like depth of removal, and change in surface profile were studied. Galvo-scanner can be used to scan a particular area by following a predefined hatch pattern. This option was used to scan samples with multiple scan patterns as shown in figure 1 and investigate whether the path followed by laser during the cleaning process affected the material properties.
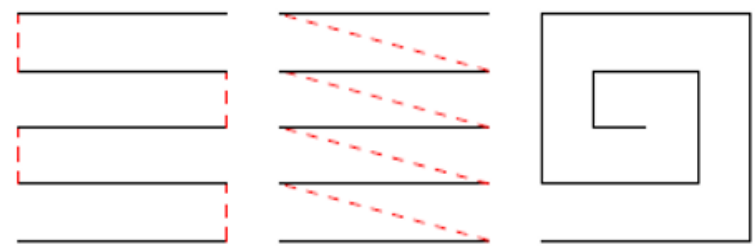

Figure 1. Paths followed by the laser beam

The change in surface roughness of the scanned area was measured with a Zeta optical profile analyser. Vickers hardness for the cleaned sample was measured and change in hardness after laser processing with different scan paths were found.

\section{Results and discussion}

The experiments were conducted for different set of speeds and different number of scans. The cleaned surface was analysed with a Zeta optical profile analyser to measure the material removal and change in surface morphology was observed.

\subsection{Effect of scan speed}

Figure 2 shows the change in depth of ablation with a change in scan speed. The experiments were conducted to study the change in surface profile when the sample is scanned with the laser at different speeds ranging from 2 $\mathrm{mm} / \mathrm{s}$ to $1000 \mathrm{~mm} / \mathrm{s}$. The lines traced were studied with a Zeta profilometer. It was observed that at lower speeds (less than $100 \mathrm{~mm} / \mathrm{s}$ ), the surface was deformed and discoloured to dark brown by the laser beam. Whereas, at speeds above $200 \mathrm{~m} / \mathrm{s}$ the surface did not show any discolouration and relatively flat surface was transformed into a wavy profile with a depression at the centre and elevations on either side after laser irradiation. The wavyness and discolouration decreases with an increase in scan speed but so does the depth of removal. The depth of removal follows a steady decrease with an increase in scan speed initially till it reaches $500 \mathrm{~mm} / \mathrm{s}$, and is observed to remain almost constant thereafter.

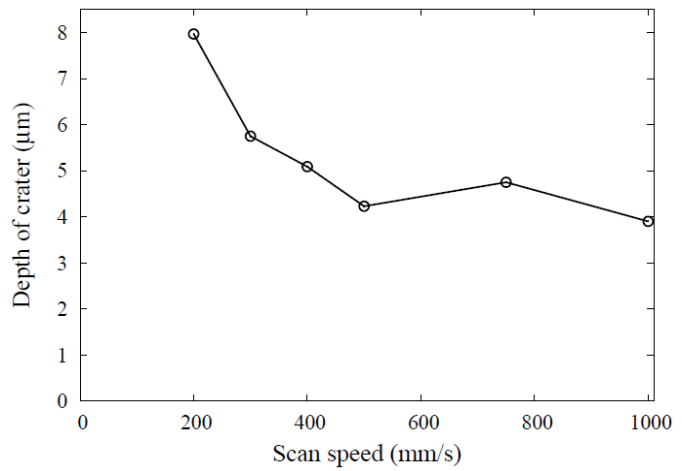

Figure 2. Depth of crater vs. scan speed

\subsection{Effect of number of scans}

Figures 3 and 4 show the surface profiles after 15 scans at $100 \mathrm{~mm} / \mathrm{s}$ and $500 \mathrm{~mm} / \mathrm{s}$, respectively. The study showed that for lower speeds, multiple scans deform the surface in a disordered way. At lower scan velocity with multiple scans, it was observed that the processed surface is irregular with peaks and valleys forming at random positions on the irradiated region (see figure 3 ).

But for higher scan speeds, the surface gets deformed into a wavy pattern with a crater in the irradiated zone and humps formed on either ends of the crater (see figure 4).

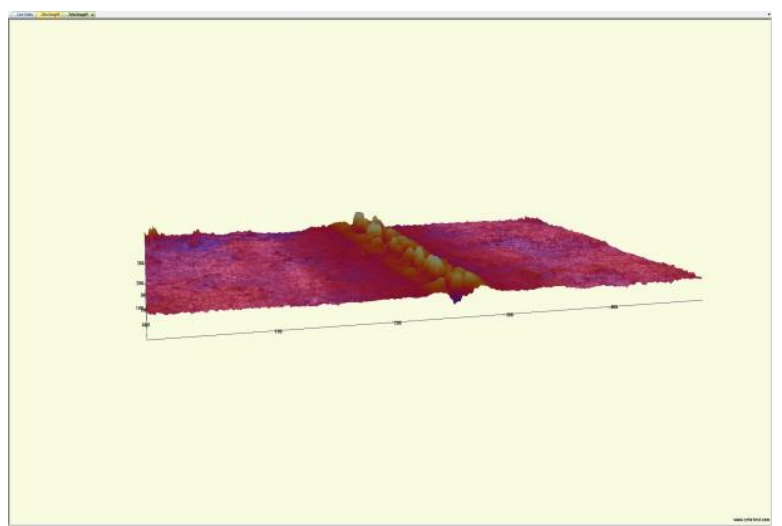

Figure 3.Surface after $15 \mathrm{scans}$ for $100 \mathrm{~mm} / \mathrm{s}$ 


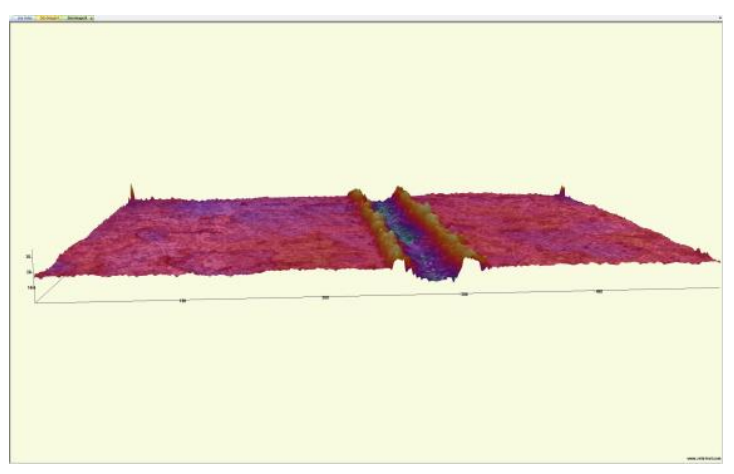

Figure 4. Surface after 15 scans for $500 \mathrm{~mm} / \mathrm{s}$

As seen in figure 4, the processed surface for high speeds can be observed to be of wavy cross section. The general wavyness of the surface remains the same for any number of passes. Figures 5 and 6 show the relationship between number of passes with the average depth of depression and the average height of peak on the surface, respectively.

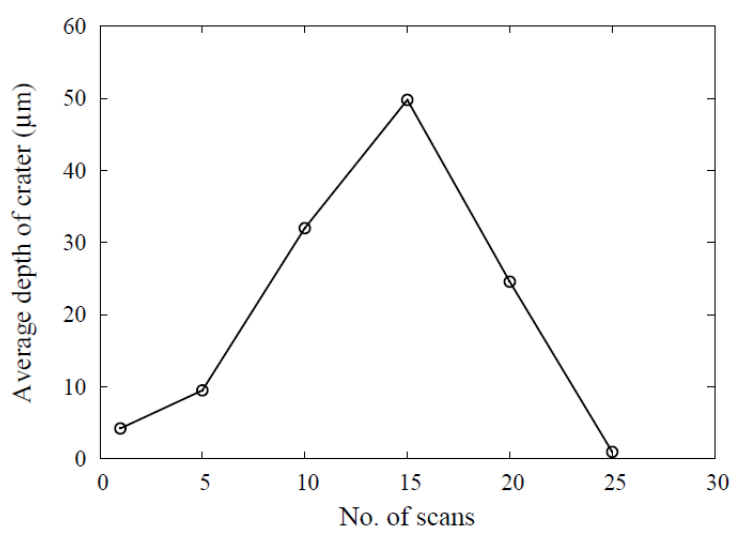

Figure 5. Average depth crater vs. number of scans

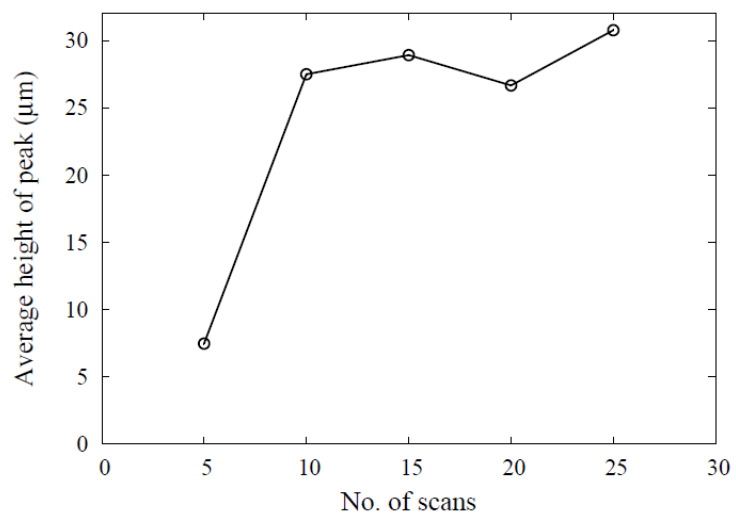

Figure 6. Average height of peak vs. number of scans

The surface shows a clear wavy profile with the peaks becoming taller with each scan for first 10 scans and remains almost same with a further increase in number of scans as seen in figure 6. But the depth of valleys (observed at central region of laser irradiated zone), increases for the first 15 scans and then decreases as seen in figure 5.

\subsection{Effect of laser power}

Unlike the scan speed, depth of removal increased with an increase in laser power or fluence (figure 7).

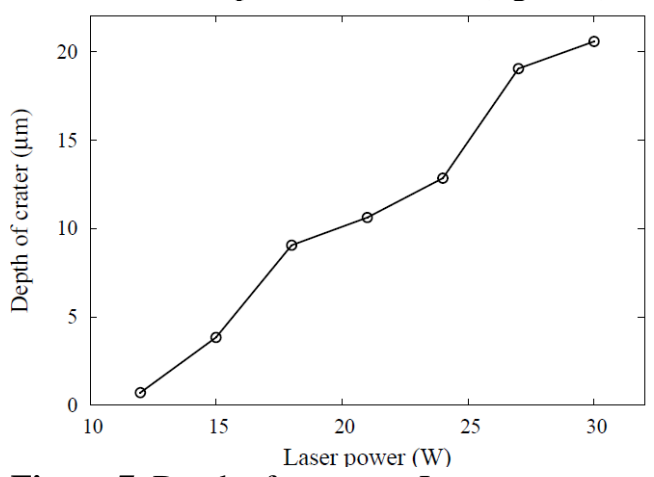

Figure 7. Depth of crater vs. Laser power.

The height of peak and depth of valley increased steadily with an increase in laser power. For lower powers the processed surface looked clean, but as power was increased the surface appeared darker after irradiation. The discolouration and change in surface topology is possibly due to a combined effect of corrosion and deformation initiated by the laser beam. Further studies can shed light on a deeper understanding of the phenomena observed during the experiments.

\subsection{Effect of scanning path}

Three scan patterns (figure 1) were selected and used to study the effect of path followed by laser in material properties of surface after inteaction with the laser. The surface was scanned once with each pattern. The study was repeated for three scans for every pattern. Each pass was timed 10 seconds apart. The roughness and hardness of this area was measured and compared with initial roughness before laser processing for rusted and pure samples.

The study showed that the surface roughness values were more or less the same irrespective of the initial condition of the sample. A pure MS surface $\left(S_{a}=0.919\right.$, $\left.\mathrm{S}_{\mathrm{z}}=6.56\right)$ and a rusted MS surface $\left(\mathrm{S}_{\mathrm{a}}=26.77, \mathrm{~S}_{\mathrm{z}}=\right.$ 346.82 ) had similar surface roughness values after cleaning. Roughness values after cleaning were $\mathrm{S}_{\mathrm{a}}=$ 1.347 to 1.463 and $S_{z}=.12 .43$ to 12.51 for pure samples. In comparison the surface roughness of the cleaned rusted samples were $S_{a}=1.413$ to 1.646 and $S_{z}=8.955$ to 12.15 . The final surface profile and surface roughness was observed to be unrelated to the initial surface profile but was dependent on scan patterns. So the surface roughness of the processed parts is found to be independent of the initial surface roughness before laser cleaning.

Surface hardness was also studied for laser cleaned surfaces. The hardness was found to have increased significantly after laser treatment. It was observed that the hardness varied according to scan path. The hardness was also diffferent in different parts of the sample scanned by a pattern. The significant change in hardness after laser processing points that the microstructure changes depend on the scan path amongst other factors. 


\section{Conclusion}

In this work, experiments were done on rusted and pure mild steel samples to study the effect of laser parameters in de-rusting process. For laser cleaning of rust there exists a range of parameters for which cleaning is satisfactory. For lower speeds and higher number of scans, the surface gets damaged by the laser. The damage seems to be a combined effect of corrosion and deformation. Further study could reveal the explicit phenomena involved. Single pass at higher speeds give a well cleaned surface. Multiple passes caused changes in surface topology which was random for low speeds and followed a particular pattern for high speeds. Path followed by laser was found to have affected the surface hardness and surface roughness of the sample. The hardness of the cleaned surface was enhanced but roughness values seemed to be independent of the initial roughness and varied only with change in laser parameters. Further studies have to be done to understand and know more about these phenomena. Precisely, the effect of scan path on the microstructure changes, and the ability to control it in order to get desired hardness values for the processed part has to be studied futher.

\section{Acknowledgments}

The authors wish to gratefully acknowledge that this research was funded by the DST-Swarnajayanti
Fellowship award [DST / SJF / ETA - 02/2014-2015] and Technology Systems Development Program [DST / TSG /AMT / 2015 / 226 / G], Government of India. The authors would also wish to thank Mrs. Bhargavi Ankamreddy for her help in conducting experiments.

\section{References}

1. X. Li, T. Huang, A. W. Chong, Opto-electronic engineering 44 340-44 (2017)

2. G. Daurelio, G. Chita, M. Cinquepalmi, Appl. Phys. A Mater. Sci. Process 69, S543-46 (1999)

3. S. N. Ali, Z. A. Taha, T. S. Mansour, Advances in Condensed Matter Physics 2014 (2014)

4. Y. F. Lu, W. D. Song, M. Hong, M. Zhong, Q. Chen, T. Chong, Jpn. J. Appl.Phys. 39 4811-13 (2000)

5. J. F. Asmus, Technology and Conservation 3 14-18 (1978)

6. Y. Koh, I. Sárady, Journal of Cultural Heritage 4 129-33 (2003)

7. P. Meja, M. Autric, P. Alloncle, P. Pasquet, R. Oltra, J. P. Boquillon Appl. Phys. A Mater. Sci. Process. 69 687-90 (1999)

8. Z. Wang, X. Zeng, W. Huang, Surf. Coatings Technol. 166 no. 1 10-16 (2003)

9. G. X. Chen, T. J. Kwee, K.P.Tan, Y.S. Choo, M. H. Hong, J. Laser Micro Nano Eng. 7 249-53 (2012) 